\title{
Avaliação da inflamabilidade de seis espécies nativas na região sul do Tocantins
}

\author{
Yasmim Andrade Ramos ${ }^{\mathrm{a}^{*}}$, Allan Deyvid Pereira da Silva ${ }^{\mathrm{b}}$, Rayna Régina Alves de Oliveira ${ }^{\mathrm{a}}$, \\ Rodrigo Araújo Fortes ${ }^{\mathrm{a}}$, Francisca de Cássia Silva da Silva ${ }^{\mathrm{a}}$, Antônio Carlos Batista ${ }^{\mathrm{b}}$, Marcos Giongo ${ }^{\mathrm{a}}$, \\ Maria Cristina Bueno Coelho ${ }^{a}$
}

\footnotetext{
${ }^{\text {a }}$ Universidade Federal do Tocantins (UFT), Brasil

${ }^{\mathrm{b}}$ Universidade Federal do Paraná (UFPR), Brasil

*Autor correspondente (yasmimaramos@gmail.com)
}

\section{N F O}

\section{Keywords \\ Cerrado \\ fire}

flammable

\begin{abstract}
A B S T R A C T
Flammability evaluation of six native species in the southern region of Tocantins.

The woody vegetation of the Cerrado presents adaptive properties to the fire. The fires during the dry season may cause significant changes in the structure and floristic composition of the vegetation. This study aimed to evaluate the flammability of six native species of a Cerrado fragment located in southern Tocantins. Samples composed of fine materials of the selected species were collected, with a diameter of less than $7 \mathrm{~mm}$, excluding young leaves and apical buds. For each species, 50 repetitions were performed, being analyzed: ignition frequency, ignition time, combustion duration, combustion index, and the determination of flammability value. Among the evaluated species, Curatella americana L., Clethra scabra Pers. and Anacardium occidentale L. Obtained significant values with emphasis on Clethra scabra Pers. Which obtained the highest mean values of the flammability variables. We also observed that most species had a combustion index classified as very high $(\mathrm{CI}=4$ and $\mathrm{CI}=5)$. With this study we can conclude that most species are flammable, and Clethra scabra Pers. Was the species that presented the best result of the flammability variables being classified as highly flammable. On the other hand, Macrolobium limbatum Spruce ex Benth. var. limbatum and Ziziphus Cinnamomum Triana \& Planch. Were the species that obtained the lowest results being classified as weakly flammable.
\end{abstract}

\section{R E S U M O}

A vegetação lenhosa do Cerrado apresenta propriedades adaptativas ao fogo. As queimadas durante a estação seca podem ocasionar em mudanças significativas na estrutura e composição florística da vegetação. Este trabalho teve como objetivo avaliar a inflamabilidade de seis espécies nativas de um fragmento de Cerrado, localizado no sul do Tocantins. Foram coletadas amostras compostas de materiais finos das espécies selecionadas, com diâmetro inferior a $7 \mathrm{~mm}$, excluindo folhas jovens e gemas apicais. Para cada espécie, realizou-se 50 repetições, sendo analisados: frequência de ignição, tempo para ignição, duração da combustão, índice de combustão, além da determinação do valor de inflamabilidade. Dentre as espécies avaliadas, Curatella americana L., Clethra scabra Pers. e Anacardium occidentale L. obtiveram valores significativos com destaque para Clethra scabra Pers. que obteve os maiores valores médios das variáveis de inflamabilidade. Observamos também que a maioria das espécies tiveram um índice de combustão classificados como muito alto ( $\mathrm{IC}=4 \mathrm{e} \mathrm{IC}=5$ ). Com este estudo podemos concluir que a maioria das espécies são inflamáveis, sendo que Clethra scabra Pers. foi a espécie que apresentou o melhor resultado das variáveis de inflamabilidade sendo classificada como altamente inflamável. Por outro lado, Macrolobium limbatum Spruce ex Benth. var. limbatum e Ziziphus cinnamomum Triana \& Planch. foram as espécies que obtiveram os menores resultados sendo classificadas como fracamente inflamáveis. 


\section{INTRODUÇÃO}

O comportamento do fogo é o resultado da interação entre clima e condições do combustível, topografia, técnica de queima e forma de ignição. As proporções da ação do fogo são essenciais para comparar queimas, para o planejamento da supressão e estimar os efeitos do mesmo (Batista, 1995). Por outro lado, essas disposições de comportamento do fogo têm sido usadas por diversos autores para representar as condições das queimas controladas em povoamentos florestais.

O Cerrado caracteriza-se por possuir estrato arbóreo muito desenvolvido, composto principalmente por indivíduos lenhosos pouco densos, no qual as copas das árvores não formam um dossel contínuo (Ribeiro e Walter, 1998). Machado et al. (2004) o descreveu como a mais rica savana do mundo do ponto de vista biológico, com uma flora estimada em cerca de 6.600 espécies.

Segundo Klink e Machado (2005), a principal forma de degradação deste bioma é a queimada intencional, provocada com o objetivo de estimular a rebrota das pastagens e para abrir novas áreas agrícolas. A vegetação lenhosa do Cerrado apresenta propriedades adaptativas ao fogo, as queimadas durante a estação seca podem ocasionar em mudanças mais significativas na estrutura e composição florística da vegetação do que as queimadas ocasionadas na estação chuvosa devida, principalmente, à intensidade delas (Coutinho, 1990; Eiten, 1994).

A vegetação deste bioma caracteriza-se por indivíduos finos e secos que são altamente inflamáveis, podendo entrar em combustão pela ação natural ou antrópica (Coutinho, 1980). Sendo que as queimadas por causas antrópicas mudaram totalmente o regime e a intensidade do fogo neste bioma (Pivello, 2011).

Barton et al. (2010) afirmam que a utilização desordenada da terra e o desenvolvimento de fronteiras agrícolas inadequadas figuram a qualidade do ambiente e consequentemente das terras exploradas, impossibilitando ações de gestão ambiental e conservação dos recursos naturais.

Podemos descrever que o conceito de inflamabilidade é composto por quatro fatores: tempo de ignição que é tempo em que aparece a primeira chama; duração da combustão que é contado a partir da primeira chama e vai até o final do fogo; combustibilidade que traduz a rapidez da queima do combustível e por último a consumibilidade que é a proporção de combustível que foi consumido pelo fogo (Anderson, 1970; Martin et al., 1994).

$\mathrm{O}$ estudo da inflamabilidade de espécies é fundamental para ajudar a traçar um perfil do comportamento do fogo, bem como manejá-lo. A aplicação das técnicas de prevenção implica na compreensão de toda a dinâmica de como ocorre o incêndio, assim como, identificar o potencial de cada uma das espécies (Neves, 2016).

Neves (2016) afirma ainda que é de suma importância se conhecer as características da vegetação e a sua associação com o fogo, e a mais utilizada desta é a determinação do grau de inflamabilidade de espécies vegetais. Com isso, este estudo teve como objetivo avaliar a inflamabilidade de seis espécies nativas de um fragmento de Cerrado, na região sul do Tocantins.

\section{MATERIAL E MÉTODOS}

O experimento foi realizado no Centro de Monitoramento Ambiental e Manejo do Fogo (CeMAF) e Laboratório de Química do Instituto Federal do Tocantins - Câmpus Gurupi, durante os dias 22 a 26 de outubro de 2018. As espécies arbóreas avaliadas foram: Macrolobium limbatum Spruce ex Benth. var. limbatum (faveira), Curatella americana L. (lixeira), Clethra scabra Pers. (carne de vaca), Ziziphus cinnamomum Triana \& Planch. (jacarandá), Anacardium occidentale L. (caju) e Tabebuia aurea Benth. \& Hook. f. ex S. Moore (caraíba). As amostras foram coletadas no fragmento de Cerrado presente no Câmpus de Gurupi da Universidade Federal do Tocantins, localizado a $11^{\circ} 44^{\prime}$ de latitude sul e $49^{\circ} 03^{\prime}$ 'de longitude oeste, a 286 metros de altitude.

\section{Coleta de dados}

A coleta das amostras ocorreu em dois dias consecutivos sem ocorrência de precipitação, evitando as condições de folhas úmidas e saturadas. As amostras eram compostas de materiais finos das espécies selecionadas, com diâmetro inferior a $7 \mathrm{~mm}$, excluindo folhas jovens e gemas apicais para evitar a variação do teor de umidade (Soares e Batista, 2007).

A manipulação do material foi realizada com o auxílio de luvas de látex e pinças num período máximo de 2 horas. Foram coletados cerca de $300 \mathrm{~g}$ de material para cada espécie arbórea com auxílio de equipamentos como facão e podão para galhos mais altos. Cerca de $100 \mathrm{~g}$ de material combustível foi destinado ao teste de inflamabilidade e duas amostras de $80 \mathrm{~g}$ de material combustível foram destinadas para determinação do teor de umidade.

Os testes de inflamabilidade foram registrados por webcam acoplada a um notebook, o que viabilizou uma análise frame a frame das gravações garantido exatidão na coleta de dados. Foram realizados 50 testes para cada espécie arbórea utilizandose um epirradiador com potência de $500 \mathrm{~W}$ (Figura 
1), garantido uma temperatura média de disco radiante em torno de $600^{\circ} \mathrm{C}$, ao fundo do epirradiador a uma distância de $32 \mathrm{~cm}$ foi fixado uma régua graduada de 1 em 1 centímetros.

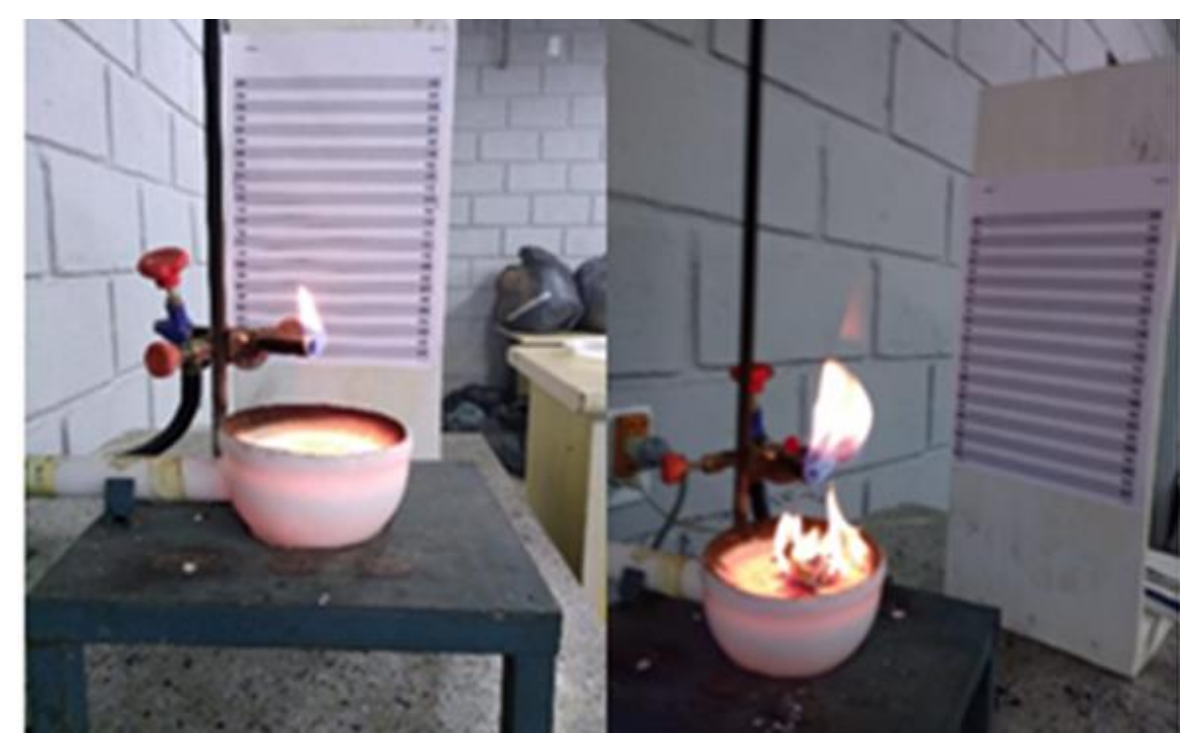

Figura 01 - Epirradiador, chama piloto e régua graduada.

Foram analisadas as seguintes variáveis no teste de inflamabilidade, conforme proposto por Petriccione (2006):

a) Tempo para ignição (TI): tempo que o material leva para iniciar a combustão;

b) Frequência de ignição (FI): número de repetições em que ocorreu ignição, considerando um TI máximo de $60 \mathrm{~s}$, em queimas que ultrapassaram este tempo foram classificadas como "queimas negativas"; c) Duração da combustão (DC): tempo que a chama se mantém acesa;

d) Índice de combustão (IC): intensidade de combustão de cada queima, sendo determinada através da média das alturas de chama e classificada segundo os índices apresentados na Tabela 1.

e) Valor de inflamabilidade (VI): atribuído de acordo com a FI e o TI (Tabela 2).

Tabela 01 - Índices do valor de combustão.

\begin{tabular}{ccc}
\hline Índice de combustão (IC) & Designação do IC & Comprimento da chama (cm) \\
\hline IC1 & Muita baixa & $<1$ \\
IC2 & Baixa & 1 a 3 \\
IC3 & Média & 4 a 7 \\
IC4 & Alta & 8 a 12 \\
IC5 & Muito alta & $>12$ \\
\hline
\end{tabular}

Tabela 02 - Índices do valor de inflamabilidade.

\begin{tabular}{ccccccc}
\hline \multirow{2}{*}{ TI $(\mathbf{s})$} & $\mathbf{2 5 - 3 8}$ & $\mathbf{3 9 - 4 1}$ & $\mathbf{4 2 - 4 4}$ & $\mathbf{4 5 - 4 7}$ & $\mathbf{4 8 - 5 0}$ \\
\cline { 2 - 7 }$>32,5$ & 0 & 0 & 0 & 1 & 1 & 2 \\
$27,6-32,5$ & 0 & 0 & 1 & 1 & 2 & 2 \\
$22,6-27,5$ & 0 & 0 & 1 & 2 & 2 & 2 \\
$17,6-22,5$ & 1 & 1 & 2 & 2 & 3 & 3 \\
$12,6-17,5$ & 1 & 1 & 2 & 3 & 3 & 4 \\
$<12,6$ & 1 & 2 & 2 & 3 & 4 & 5 \\
\hline
\end{tabular}

Em que: FI - frequência de ignição e TI - tempo para ignição. O valor de inflamabilidade (VI) será classificado em função do número correspondente, em que: VI = 0 (fracamente inflamável); VI = 1 (pouco inflamável); VI = 2 (moderadamente inflamável); VI = 3 (inflamável); VI = 4 (altamente inflamável) ou VI = 5 (extremamente inflamável). 
O material utilizado nas repetições era recém-coletado com teor de umidade variável, devido as características da espécie e do estágio de desenvolvimento do indivíduo. Para o teste de inflamabilidade foram utilizadas amostras de aproximadamente $1 \pm$ $0,1 \mathrm{~g}$ sendo exposta ao epirradiador e à chama piloto, determinando-se a FI de acordo com o número de vezes que a amostra entrava em ignição até ser consumida pela chama. Ao final de todo teste contabilizou-se o tempo total para eliminação da amostra, ocorrendo ou não a ignição do material.

Com a análise das gravações determinou-se a Altura da Chama (AC), ponto máximo que a chama atingiu durante a combustão (Valette, 1992). A partir da AC foi possível determinar o IC pela Tabela 01. Procurando na tabela 2 os valores de FI e TI, determinamos o índice de inflamabilidade da amostra, que vai de 0 a 5 (Tabela 02). Tal índice indica o quanto a espécie pode ser inflamável, no valor 0 a espécie é considerada fracamente inflamável e no valor 5 é considerada extremamente inflamável (Valette, 1992).

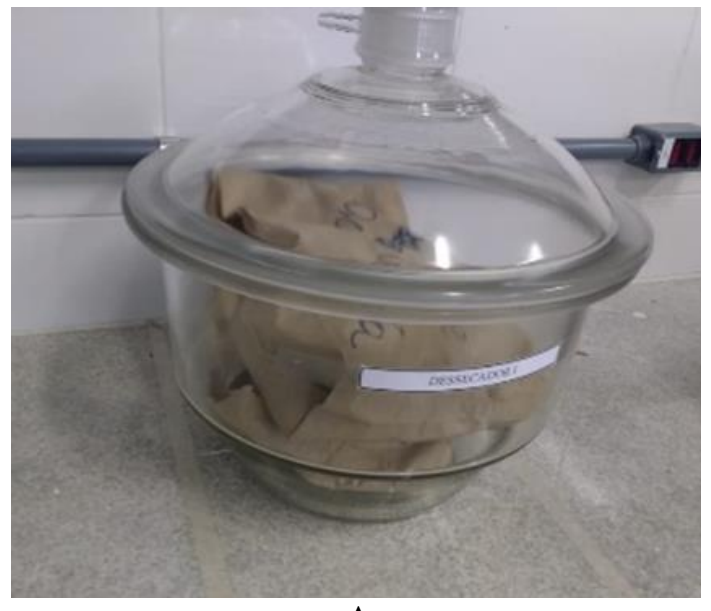

A
Para a determinação do teor de umidade inicial da amostra (TU) adotou-se a equação descrita na NBR 7190 que corresponde à relação entre a massa da água contida na amostra e a massa da amostra seca. Duas amostras de $80 \pm 0,1 \mathrm{~g}$ de material combustível foram utilizadas para determinação do teor de umidade, sendo pesadas acondicionadas em saco de papel kraft identificados e secas em estufa a uma temperatura de $75^{\circ} \mathrm{C}$ por 48 horas, sendo determinadas as massas em temperatura ambiente após secagem (Figura 02).

$$
T U(\%)=\frac{m i-m s}{m s} * 100
$$

Onde:

TU é o teor de umidade inicial, em percentual; mi é a massa inicial, em gramas; ms é a massa seca, em gramas.

Figura 02 - Determinação do teor de umidade das amostras. Dessecador utilizado para resfriamento das amostras (A), determinação da massa do material seco (B).

Procedeu-se a análise de variância (ANOVA) para cada espécie. Foi utilizado o teste de Tukey ao nível de 5\% de probabilidade para comparação entre médias, envolvendo todas as espécies, os cálculos estatísticos foram feitos pelos Softwares SISVAR e Excel 2010.

\section{RESULTADOS E DISCUSSÃO}

Analisando os resultados, podemos observar que as espécies Curatella americana L., Clethra scabra Pers. e Anacardium occidentale L. obtiveram valo-

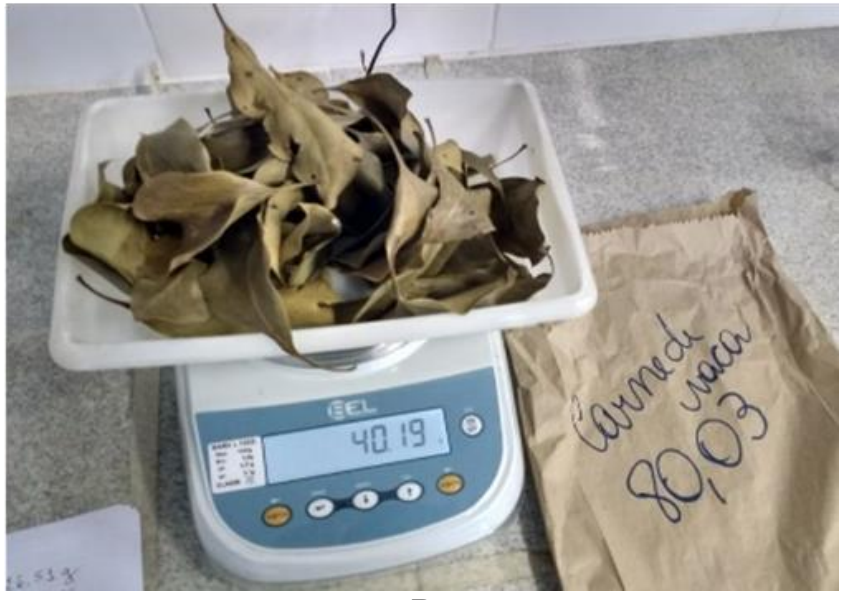

B res significativos com destaque para Clethra scabra Pers. que obteve os maiores valores médios das variáveis de inflamabilidade conforme apresentado na tabela 3.

A espécie Macrolobium limbatum Spruce ex Benth. var. limbatum não obteve resultados significativos para nenhuma das variáveis estudadas. Petriccione (2006) ao estudar a inflamabilidade de diversas espécies do mediterrâneo também não encontrou resultados significativos para Robinia pseudoacacia espécie esta pertencente a mesma família botânica de M. limbatum Spruce ex Benth. var. limbatum, Fabaceae. 
Ainda com os resultados da tabela 3 observamos que a espécie Ziziphus cinnamomum Triana \& Planch da família Rhamnaceae, teve resultados quase que semelhantes aos da espécie citada anteriormente, mesmo sendo estas de famílias diferentes. Não foram encontrados estudos para espécies da mesma família ou gênero para comparativos.

Tabela 03 - Valores médios das variáveis de inflamabilidade.

\begin{tabular}{ccccccc}
\hline \multirow{2}{*}{ Nome comum } & \multirow{2}{*}{ Espécie } & \multicolumn{3}{c}{ Média } & \multirow{2}{*}{ U\% } & \multirow{2}{*}{ FI } \\
\cline { 2 - 5 } & Macrolobium limbatum & $0,0 \mathrm{a}$ & $0,0 \mathrm{a}$ & $0,0 \mathrm{a}$ & $\mathbf{1 6 5 , 5 0}$ & $\mathbf{0}$ \\
Faveira & Curatella americana & $7,16 \mathrm{a}$ & $2,03 \mathrm{a}$ & $8,85 \mathrm{a}$ & $\mathbf{1 9 2 , 4 6}$ & $\mathbf{5 6}$ \\
Lixeira & Clethra scabra & $250,49 \mathrm{a}$ & $8,22 \mathrm{a}$ & $19,38 \mathrm{a}$ & $\mathbf{1 0 1 , 5 0}$ & $\mathbf{1 0 0}$ \\
Carne de vaca & Ziziphus cinnamomum & $0,18 \mathrm{a}$ & $0,03 \mathrm{a}$ & $0,33 \mathrm{a}$ & $\mathbf{3 2 2 , 3 4}$ & $\mathbf{2}$ \\
Jacarandá & Anacardium occidentale & $10,47 \mathrm{a}$ & $6,19 \mathrm{a}$ & $15,40 \mathrm{a}$ & $\mathbf{1 6 0 , 2 9}$ & $\mathbf{5 0}$ \\
Caju & Tabebuia aurea & $17,35 \mathrm{a}$ & $6,93 \mathrm{a}$ & $17,73 \mathrm{a}$ & $\mathbf{1 9 6 , 2 8}$ & $\mathbf{9 0}$ \\
Caraíba & &
\end{tabular}

Em que TI: tempo de ignição; DC: duração da combustão; AC: altura da chama; U\%: umidade relativa, em percentagem; FI: frequência de ignição.

Todas as variáveis relacionadas com o fogo estão fortemente ligadas com o teor de umidade dos materiais combustíveis, sobretudo o tempo de ignição e o comprimento das chamas (Hernando, 2009). Alessio et al. (2008) descreveu que podem existir outros fatores nas espécies que possam influenciar diretamente o teor de umidade dos materiais combustíveis como a presença de terpenos voláteis.

Observamos que todas as espécies apresentaram teor de umidade acima de $100 \%$ (tabela 03) e $\mathrm{Zi}$ ziphus cinnamomum Triana \& Planch. obteve um resultado muito elevado (322,34\%) o que pode explicar seus baixos valores médios das variáveis de inflamabilidade.

Porém, ao avaliar a inflamabilidade de cinco espécies de floresta ombrófila mista no Paraná, Neves (2016) descreveu que a diferença nos teores de umidade das espécies pode estar relacionada ao estado fisiológico das espécies e ao comportamento na manutenção de suas folhas.

Para este estudo podemos considerar ainda, que as amostras foram coletadas no mês de outubro (período de chuvas no estado do Tocantins), o que eventualmente explicaria esse alto teor de umidade em todas espécies estudadas. Rodriguez et al. (2016) afirmou que a umidade do material em análise é de extrema importância para a interpretação dos resultados.

$\mathrm{Na}$ avaliação da inflamabilidade de espécies para uso em cortina de segurança no sul do Brasil, Batista et al (2012) observaram a correlação entre o teor de umidade e as variáveis de combustão. Segundo os mesmos autores, a umidade da vegetação é um importante fator na ignição e na propagação do fogo.

Foram analisados o índice de combustão (IC) e o valor de inflamabilidade (VI) das espécies estudadas (Tabela 04). Nessa tabela podemos observar que somente duas espécies (Macrolobium limbatum Spruce ex Benth. var. limbatum e Ziziphus cinnamomum Triana \& Planch.) obtiveram um valor de inflamabilidade " 0 ", ou seja, fracamente inflamáveis. O que mais uma vez podemos explicar pelo alto teor de umidade nas duas espécies.

Anacardium occidentale L. e Tabebuia aurea Benth. \& Hook. f. ex S. Moore, apesar de serem de famílias diferentes, tiveram os mesmos valores de IC e VI, sendo classificadas como sendo inflamáveis. Já Clethra scabra Pers. obteve um valor de VI $=4$, sendo classificada como uma espécie altamente inflamável. Não foram encontrados nas literaturas resultados próximos a família nem ao gênero desta para poder comparar os resultados.

Tabela 04 - Índice e combustão (IC) e valor de inflamabilidade (VI).

\begin{tabular}{lcc}
\hline \multicolumn{1}{c}{ Espécie } & IC & VI \\
\hline Macrolobium limbatum Spruce ex Benth. var. limbatum & 1 & 0 \\
Curatella americana L. & 4 & 2 \\
Clethra scabra Pers. & 5 & 4 \\
Ziziphus cinnamomum Triana \& Planch. & 1 & 0 \\
Anacardium occidentale L. & 5 & 3 \\
Tabebuia aurea Benth. \& Hook. f. ex S. Moore & 5 & 3 \\
\hline
\end{tabular}


Pela análise da tabela 04, observamos que a maioria das espécies tiveram um índice de combustão classificados como muito alto (IC $=4$ e IC $=5$ ), com uma altura média da chama acima de $12 \mathrm{~cm}$, conforme a metodologia de Valete (1990).

\section{CONCLUSÕES}

Com este estudo podemos concluir que a maioria das espécies são inflamáveis, sendo que Clethra scabra Pers. foi a espécie que apresentou o melhor resultado das variáveis de inflamabilidade sendo classificada como altamente inflamável. Por outro lado, Macrolobium limbatum Spruce ex Benth. var. limbatum e Ziziphus cinnamomum Triana \& Planch. foram as espécies que obtiveram os menores resultados sendo classificadas como fracamente inflamáveis. Talvez isso tenha ocorrido pelo alto teor de umidade que foram encontradas nestas espécies.

\section{REFERÊNCIAS BIBLIOGRÁFICAS}

ANDERSON, H.E. (1970). Forest fuel ignitibility. Fire Technology 6, p.312-322.

BARTON, C.M.; ULLAH, I.I.; BERGIN, S. Land use, water and Mediterranean landscapes: modelling long-term dynamics of complex socio-ecological systems. Philosophical Transactions da Royal Society, v.368, p.5275-5297, 2010.

COUTINHO, L.M.O Cerrado e a ecologia do fogo. Ciência Hoje, v.12, n.68, p.22-30, 1990b.

COUTINHO, L.M. Fire in the ecology of Brasilian Cerrado. In GOLDAMMER, J.G., (Ed.) Fire in the Tropical Biota: ecological processes and global challenges. Ecological Studies. Berlin: Springer-Verlang, 1990. p.82-105.

EITEN, G. Vegetação do cerrado. In: PINTO, M.N. (Org). Cerrado: caracterização, ocupação e perspectivas. Brasília: Edunb/SEMATEC, 1994. p.17-73.

HERNANDO, C.L. Combustibles forestales: inflamabilidad. In: Vélez, R. M. (Coord). La defensa contra incêndios forestales: fundamentos y experiências, 2. ed. Espanha: Mcgrawhill, 2009.

KLINK, C.A. MACHADO, R.B.A conservação do Cerrado brasileiro. Megadiversidade. v.1, n.1. Julho 2005.

MARTIN, R.E.; GORDON, D.A.; GUTIERREZ, M.E; LEE, D.S; MOLINA, D.M.; SCHROEDER, R.A.; SAPSISDB STEPHENS, S.L.; CHAMBERSM. (1994). Assessing the flammability of domestic and wildland vegetation. In 'Proceedings of the 12th 60 conference on fire and forest meteorology', (Society of American Foresters: Bethesda, MD), Jekyll Island, GA. p. 130-137

NEVES, P.C. Avaliação da inflamabilidade de cinco espécies da floresta ombrófila mista. 2016.

PETRICCIONE, M. Infiammabilità della lettiera di diverse specie vegetali di ambiente mediterraneo. 2007. Tese de Doutorado. Università degli Studi di Napoli Federico II.
SOARES, R.V.; BATISTA, A.C.; Tetto, A.F. Incêndios florestais: controle, efeitos e uso do fogo. 2. ed. Curitiba, 2017. 255p.

PIVELLO, V.R.; COUTUNHO, L.M. The use of fire in the cerrado and amazonian rainforests of brazil: past and present. Fire ecology, 2011. v.7, n.1, p.24-39.

RIBEIRO, J.F.; WALTER, B.M.T. Fitofisionomias do bioma cerrado. In: SANO, S. M.; ALM EIDA, S. P. (Eds.). Cerrado: ambiente e flora. Planaltina: Embrapa Cerrados, 1998. p.89-166.

RODRIGUEZ, Y.C. et al. Inflamabilidad de especies vegetales del ecosistema de pinares. Revista Cubana de Ciencias Forestales: CFORES, v.4, n.1, p.5, 2016.

VALETTE, J.C. Inflammabilities of mediterranean species. Porto Carras: Research and Development of the European Commission European School of Climatology and Natural Hazards, 1992. 12 p. (Document PIF9208). 